\title{
Ipsilateral Acute Monteggia Lesion and Malunion of Galeazzi Fracture in an Adult. A Case Report
}

\author{
JBS Essoh, MD, DK Aka, MD, LS Bada, MD
}

Department of Orthopaedic Surgery, Yopougon University Teaching Hospital, Abidjan, Côte d'Ivoire

\section{ABSTRACT}

Presentation of Monteggia and Galeazzi fractures in the same limb is a rare injury. We report an unusual case in which an acute Monteggia fracture was associated with a malunited Galeazzi fracture in an adult patient. The mechanism of this complex injury is discussed.

\section{Key Words:}

Fracture-dislocation, Galeazzi Fracture, Malunion, Monteggia fracture

\section{INTRODUCTION}

Monteggia and Galeazzi fracture-dislocations are complex injuries of the upper limb ${ }^{1}$. An ipsilateral combination of these injuries is an exceedingly rare occurrence ${ }^{2}$. We report

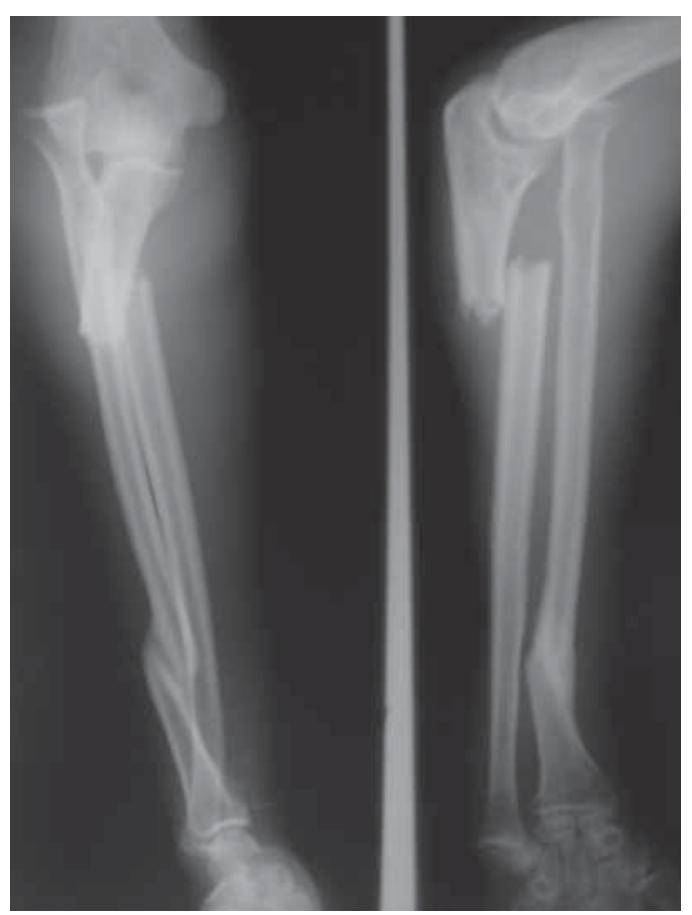

Fig. 1: Radiograph showing ipsilateral acute Monteggia lesion and a malunion of Galeazzi fracture. here a case in which an acute Monteggia fracture was associated with a malunited Galeazzi fracture in an adult patient, drawing special attention to the mechanism of injury.

\section{CASE REPORT}

A 28-year-old right-handed male taxi driver that attempted to take a curve at a high speed and overturned. On presentation at the hospital, he was conscious and complained of left forearm pain. He could only remember that his left upper limb was trapped in the car. Examination showed a painful swelling of the elbow and the upper region of the forearm. There was no neurovascular involvement. Radiographic examination revealed an acute Monteggia lesion (fracture of third proximal ulna shaft associated with anterior dislocation of the radial head), and a malunited radial shaft (distal 1/3) fracture with

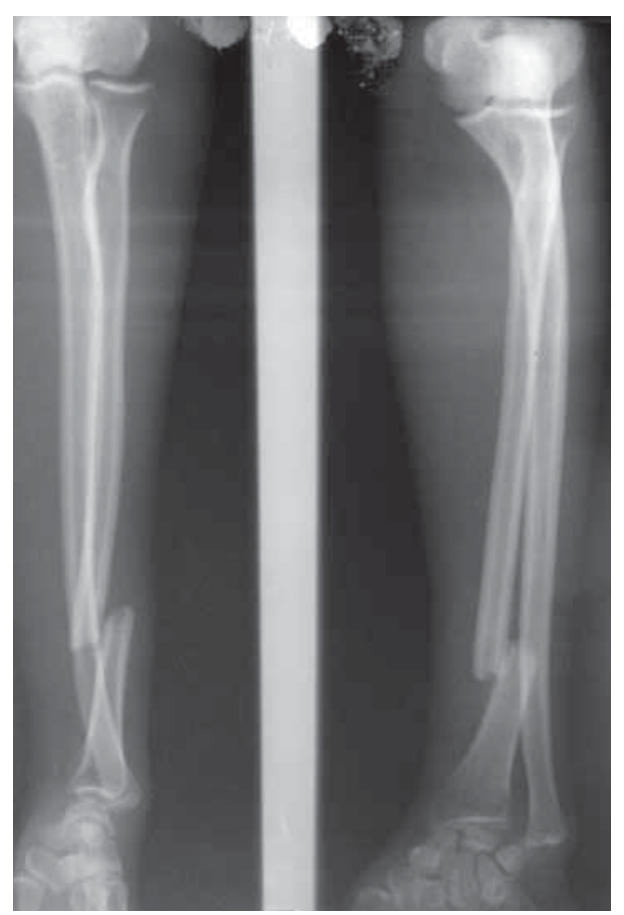

Fig. 2: Radiograph showing Galeazzi fracture in the same patient three years before. 
dislocation of the distal radioulnar joint (Figure 1). Three years previous to this accident, the patient had sustained a Galeazzi fracture of the left forearm (Figure 2) caused by a fall on an outstretched arm. This lesion was treated by traditional bonesetters. Malunion occurred but disability as a result of this condition did not prevent him from driving. As a result, for this second episode of fracture, the patient did not accept the surgical treatment proposed. He withdrew from hospital and went to a traditional practitioner for assistance.

\section{DISCUSSION}

According to the patient, the functional outcome following the Galeazzi fracture of the left forearm was satisfactory such that he was able to resume his work as a driver. However, the anatomical situation may have been unstable and with limited range of motion; it could have precipitated the recent fracture-dislocation. It is well known that the normal function and biomechanics of the forearm requires that both bones have normal length and anatomy ${ }^{3}$.

Importantly, there is a close relationship between the radius and the ulna and injury to either bone will have a direct impact on the other. When one of these bones suffers a significant trauma, such as displaced or angulated fracture, it is highly likely that the other will be affected, usually at one or both of the radioulnar joints ${ }^{4}$. Specific mechanism in the patient described here is likely akin to that described by Yasutomi et al in the setting of an anterior dislocation of the radial head secondary to malunion of the forearm bones ${ }^{5}$. They reported that an eccentric load to the joint in the case of malunion of the forearm would lead to the dislocation of the radial head. Late degenerative disease of the elbow and the wrist seen in malunion of forearm is ascribed to excess load applied to the joints ${ }^{3}$. For the patient described here, it did not seem reasonable to exclude such conditions at the elbow joint even though it appeared to be normal on plain radiographs. A load-bearing radial head is unstable and prone to dislocation at the slightest injury. We suggest that load exerted on the radial head creating instability is a leading cause of dislocation, with the fracture of the ulna being the trigger.

This case supports the view that attention should be paid to both Monteggia and Galeazzi lesions, as they are unstable injuries. A patient with one of these injuries is not necessarily spared from the other injury and subsequent treatment may be very difficult. 


\section{REFERENCES}

1. Simpson NS, Jupiter JB. Complex fracture patterns of the upper extremity. Clin Orthop 1995; 318: 43-53.

2. Clare DJ, Corley FG, Wirth MA. Ipsilateral combination Monteggia and Galeazzi injuries in an adult. J Orthop Trauma 2002; 16: $130-4$

3. Richards RR. Current concepts review. Chronic disorders of the forearm. J Bone Joint Surg 1996; 78A: 916-30.

4. Perron AD, Hersh RE, Brady WJ, Keats TE. Orthopedic pitfalls in the ED: Galeazzi and Monteggia fracture-dislocation. Am $J$ Emerg Med 2001; 19: 225-8.

5. Yasutomi T, Nakatsuchi Y, Koike H. Anterior dislocation of the radial head secondary to malunion of the forearm bones. $J$ Shoulder Elbow Surg 2000; 9: 536-40. 